\title{
Les villes artificielles comme espaces de formation de l'ordre politique : l'entrainement aux scénarios apocalyptiques des polices européennes
}

\section{Andrea Kretschmann}

\section{CpenEdition}

\section{Journals}

Édition électronique

URL : https://journals.openedition.org/cdg/7154

DOI : $10.4000 /$ cdg. 7154

ISSN : 2107-7266

Éditeur

UMR 245 - CESSMA

Référence électronique

Andrea Kretschmann, « Les villes artificielles comme espaces de formation de l'ordre politique l'entrainement aux scénarios apocalyptiques des polices européennes », Carnets de géographes [En ligne], 15 | 2021, mis en ligne le 30 avril 2021, consulté le 28 mai 2021. URL : http:// journals.openedition.org/cdg/7154; DOI : https://doi.org/10.4000/cdg.7154

Ce document a été généré automatiquement le 28 mai 2021.

\section{(†) $\odot$}

La revue Carnets de géographes est mise à disposition selon les termes de la Licence Creative Commons Attribution - Pas d'Utilisation Commerciale - Pas de Modification 4.0 International. 


\title{
Les villes artificielles comme espaces de formation de l'ordre politique : l'entrainement aux scénarios apocalyptiques des polices européennes
}

\author{
Andrea Kretschmann
}

1 Depuis quelques décennies déjà, les polices d'Europe assurent plus que le contrôle et la régulation des seuls espaces urbains : elles contrôlent également des « espaces autres » (Foucault, 1984), dans lesquels, selon la conception de Foucault de l'hétérotopie, il existe des règles propres, mais qui reflètent les relations sociales d'une manière particulière. Ces espaces construits par les polices elles-mêmes sont destinés à la formation des agents. Ils simulent des paysages urbains sans habitants, des villesfantômes. Ces infrastructures construites par les administrations policières entrainent aux manœuvres de la police des foules, c'est-à-dire à la maîtrise de situations telles que des matchs de football et autres événements de cette ampleur, mais aussi bien sûr des manifestations. Les polices y développent des scénarios censés se rapprocher le plus possible des conditions rencontrées sur le terrain ${ }^{1}$, scénarios qu'elles s'emploient ensuite à traduire en termes d'engagement matériel et humain. Dans un jeu de rôles interchangeable, les uns ${ }^{2}$ endossent le rôle de manifestants, les autres d'intervenants secondaires (des journalistes par exemple), alors que d'autres encore jouent celui des policiers. Les manifestations sont ainsi simulées avec parfois plusieurs centaines de policiers. La plupart du temps, ces événements simulés donnent lieu à des affrontements sérieux: jets de bouteilles ou de cocktails-molotov sur la police, incendies de véhicules, construction de barricades. Au cours de ces entraînements, les polices simulent principalement les situations perçues comme antagonistes ${ }^{3}$ qu'elles $^{\prime}$ peuvent vivre au cours d'événements protestataires comme, par exemple, la présence 
de contre-manifestants ou les actions organisées par des mouvements écologistes ou des syndicats.

Tout comme leur équivalent pour l'armée, les villes policières artificielles se multiplient à l'échelle de la planète (Bialasiewicz, et al., 2007; Graham, 2011; Kretschmann, 2016). La plus grande ville artificielle d'Europe est aujourd'hui le Centre national d'entraînement des forces de gendarmerie (CNEFG) à Saint-Astier (Dordogne). Ce centre fut érigé par la Gendarmerie nationale et plus précisément la Gendarmerie mobile dans les années 1970 (Berlière, Lévy 2011 : 242). Il est considéré comme un modèle du genre en Europe et dans le monde (Bruneteaux, 1996; Goreau-Ponceaud, Ponceaud-Goreau, 2014). Le centre d'entrainement des CRS de la Police nationale à Clermond-Ferrand dispose d'un espace de formation comparable, mais déployé sur un espace beaucoup plus restreint. De telles villes ont également été construites ailleurs en Europe, en Allemagne, en Angleterre ou en Irlande du Nord. La mise en place de ces infrastructures d'entraînement sous forme de villes artificielles fait suite à divers " traumatismes » policiers causés par des échecs sur le terrain, couplés à des moments de crise de légitimité de la police vis-à-vis du public (pour la France, voir GoreauPonceaud, Ponceaud-Goreau, 2014 : 2f).

3 A la fin des années 1960, les polices en France et en Allemagne ont en effet été confrontées à des mobilisations massives, dans un contexte marqué par l'élargissement de la compréhension du concept de "démocratie» (Fillieule, Jobard, 2020 : 39-44). L'Allemagne fut marquée par de grandes manifestations étudiantes; en 1967, un étudiant fut abattu par un policier. En France, les manifestations d'étudiants et la grève générale de 1968 durèrent des semaines et donnèrent lieu à de violents affrontements avec la police. Dans les années 1980, la police britannique a fait face à ce que l'on a appelé des "émeutes urbaines» (Brion, Rea 1992) en pleine montée des tensions sociales entre populations immigrées et une police structurellement raciste, provoquée par la néo-libéralisation de la politique économique et l'apparition simultanée d'une récession mais aussi par une politique favorisant la confrontation avec les immigrants, les minorités ethniques, les bénéficiaires de l'aide sociale et les syndicats, (Waddington 1994 : 27). Dans ces trois pays, la gestion des évènements par les forces de l'ordre, jugée brutale et disproportionnée, a été vivement critiquée, ce qui a entrainé une importante crise de légitimité des forces de police.

4 Les polices entrent alors dans une phase de réflexion ouverte et expérimentent de nouvelles approches de gestion des foules (Berlière, Lévy $2011: 242$ ). C'est dans cette perspective que sont développées des simulations plaçant des manifestants et des policiers dans un environnement urbain fictif, afin de mettre en place des processus de désescalade et de gestion différentielle ou négociée des mobilisations. La France ouvre son centre de formation à la fin des années 1960, l'Angleterre au milieu des années 1980 et l'Irlande du Nord à la fin des années 1990. En Allemagne, il n'y a, au grand regret de nombreux policiers, pas de centre spécifique de formation au maintien de l'ordre, mais certains modules d'enseignement prévoient des simulations de manifestations.

5 Le présent article s'appuie sur une recherche ethnographique associant des périodes d'observations sur les sites d'entrainement, des entretiens et l'analyses de documents (voir par exemple DeVault, McCoy, 2012) pour étudier ces espaces urbains fictifs dans quatre contextes nationaux différents ${ }^{4}$. Au total, nous avons mené plus de 50 entretiens avec des policiers (formateurs et participants à la formation de base, effectués principalement dans la langue du pays et en nombre similaire dans chaque pays ${ }^{5}$ ) et un 
total de huit semaines d'observations (réparties à peu près également entre tous les pays). Dans ce contexte, de nombreux documents internes à la police ont également été pris en compte (plans de formation, documents de formation, etc.). Tous les résultats suivants, sauf référence à d'autres travaux, sont tirés de l'analyse de ces données. Tous les noms des interlocuteurs ont été anonymisés.

6 Partant de l'hypothèse que les polices contribuent à façonner les espaces de l'ordre politique, ce que Goreau-Ponceaud et Ponceaud-Goreau (2014) avaient déjà formulé à propos de la gendarmerie mobile française, mon étude interroge la manière dont ces entrainements contribuent à construire l'espace «réel» une fois transposés à l'extérieur du monde artificiel où ils se déroulent. A partir de l'observation empirique des différentes rationalités organisant ces entrainements dans quatre pays européens, je mets en lumière leur possible impact sur les évènements protestataires en Europe et la façon dont les enseignements dispensés au cours de ces simulations peuvent affecter les opérations de maintien de l'ordre. Nous faisons l'hypothèse que les simulations sont un instrument particulièrement puissant pour modeler la réponse policière et, en conséquence, pour construire la réalité sociale.

7 Jusqu'à maintenant seules deux études de cas sous forme de courts articles étaient disponibles ${ }^{6}$. Pour combler le manque de connaissances vis-à-vis de ces installations et de ce qui s'y déroule, cet article met principalement l'accent sur les caractéristiques communes de ces formations de différentes polices nationales : il vise à saisir la logique générale à l'œuvre derrière la conception de ces espaces de simulation et leurs effets sur le maintien de l'ordre. En cela, il s'inscrit dans un cadre de recherche hérité de la sociologie de la ville appliquée à la police, qui défend l'hypothèse selon laquelle l'action policière est toujours spatialement située mais que ces éléments spatiaux sont toujours «profondément imbriqués» avec des éléments culturels et sociaux (Herbert, 1997: 21, voir aussi Lambert, 2016). Herbert remarque ainsi, à propos des patrouilles ordinaires de police, qu'« en s'efforçant de faire respecter les notions socialement construites d'ordre public, les agents définissent et cherchent à contrôler les espaces qu'ils patrouillent " (ibid.). Il est fréquemment observé dans la littérature que ces rondes policières entrainent une criminalisation de l'espace lui-même (Belina, Wehrheim, 2011 : 211), c'est-à-dire que l'espace même devient l'objet du soupçon, impliquant tous les acteurs qui s'y trouvent (Kretschmann, Legnaro, 2019). Une telle criminalisation des espaces urbains découle d'une vision déterministe qui ignore les conditions de vie et les rapports sociaux au profit d'une personnalisation de l'espace: celui-ci est alors considéré comme déclenchant inéluctablement certaines formes de criminalité (Belina, Werheim, 2011).

8 Je montre ainsi dans cet article que les villes artificielles (dans leur matérialité et dans leur utilisation) renforcent les dynamiques policières de criminalisation des espaces, par le biais de la mise en scène de mouvements sociaux eux-mêmes systématiquement criminalisés. Cette dynamique de construction revêt un sens littéral, dans la mesure où les polices ne s'approprient pas symboliquement un lieu qui leur pré-existe, mais le construisent aux fins mêmes d'y représenter des actes protestataires considérés en grande majorité comme dangereux, illégitimes ou illégaux. Or, le maintien de l'ordre est «irréductiblement politique» (Waddington, 1996: 129), ce qui signifie que la conception des espaces de formation de la police ainsi que l'utilisation de ces espaces, ont une influence certaine sur les conditions dans lesquelles l'ordre politique est établi, 
et jouent aussi un rôle, nous le verrons, en tant qu'espaces d'expérience et de contrôle des émotions.

Dans une première partie, nous présentons la démarche empirique et développons la méthode employée au cours de l'enquête. Dans un deuxième temps, nous abordons spécifiquement les espaces construits afin de permettre ces entraînements. Nous montrons dans une troisième partie comment la forme de ces espaces fait également d'eux des espaces d'expérience. La partie suivante montre comment ces espaces sont conçus comme des "géographies assaillantes" (Crank, 2004: 161). La cinquième et dernière partie permet de traiter de la puissance socialement constituée de l'entraînement et tente de la replacer dans le contexte actuel du maintien de l'ordre.

\section{Objet empirique et méthode : la ville artificielle comme lieu d'entrainement des polices européennes}

10 Ma recherche s'appuie sur une étude, menée à l'échelle européenne, sur les programmes de formation continue au maintien de l'ordre au Royaume Uni, en France, en Allemagne et en Irlande du Nord de 2015 à 2019. En Angleterre, j'ai enquêté au Metropolitan Police Service Specialist Training Centre (MPSTC) à Gravesend dans le Kent, qui est un centre accessible à la Metropolitan Police Service, au British Transport Service, à la London City Police et à l'occasion aux polices du Kent et du Sussex. En Irlande du Nord, l'enquête a été menée au Public Order Tactical Village près de Belfast, lequel accueille le Police Service d'Irlande du Nord. En France, j'ai observé les entraînements proposés au Centre national d'entraînement des forces de gendarmerie (CNEFG) à Saint-Astier en Dordogne. En Allemagne, où n'existe pas de ville artificielle d'entrainement, j'ai enquêté auprès de la police de Basse-Saxe, au centre de formation continue de Lüchow, dans la région du Wendland. Ces cas d'étude ont été sélectionnés pour tenir compte des différentes traditions particulières en matière de maintien de l'ordre en Europe. Chaque police (à l'exception de l'Allemagne) dispose d'un seul grand centre de formation de type ville artificielle. Celui-ci se trouve généralement dans une petite ville ou une zone rurale et comprend des salles de classe, des bureaux, des dortoirs, une cantine, etc. Ces lieux sont différents des villes artificielles militaires, même si les forces de police s'entraînent occasionnellement dans les centres d'entrainement de l'armée. Seules les forces de police viennent s'entrainer dans les centres de formation sur lesquels nous avons enquêté.

11 Ces espaces présentent quelques-unes des caractéristiques typiques d'une ville (les zones rurales sont rarement représentées, à l'exception du centre d'entrainement français qui, grâce à son bon financement, peut se permettre d'avoir également une zone rurale) : des bâtiments administratifs, des stations-services, des commerces, des espaces publics. Parfois, divers quartiers distincts sont représentés, comme un centreville ou un quartier administratif. Malgré toutes leurs propriétés urbaines, ces lieux ne sont pas à proprement parler habités : ils sont dépourvus d'habitants, ne sont pas accessibles au public, ils ne sont visibles sur aucune carte routière et ne sont soumis à aucun pouvoir administratif. Leurs existences se déploient sous les radars, comme des espaces planifiés sur lesquels rien ne pousse et où rien n'est laissé au hasard. De temps à autre, des scénographies les investissent pour donner à voir différentes situations de maintien de l'ordre : attaque terroriste, émeute, manifestation. 

maintien de l'ordre dans le cadre de formations initiales et continues. Cette étude se concentre sur la formation initiale qui représente la plus grande partie de l'activité des centres, mais qui recouvre des réalités différentes d'un pays à l'autre et d'une organisation policière à l'autre. Si la formation initiale continue au maintien de l'ordre dans la police nord-irlandaise ne dure qu'un jour, et deux jours dans la police londonienne, elle dure en revanche 15 jours chez les gendarmes français. En Angleterre et en Irlande du Nord, il n'y a pas d'unités de police dédiées exclusivement au maintien de l'ordre qui s'y consacreraient à plein temps. En Allemagne, où l'on trouve des unités de police spécialisées sur le maintien de l'ordre (Bereitschaftspolizei), il n'y a pas de standards homogènes à l'échelle fédérale dans la formation des agents au maintien de l'ordre, à l'inverse de ce que l'on observe en France ou en Angleterre. La formation de base au maintien de l'ordre se déroule par petits groupes de cinq à trente personnes au maximum, sous la responsabilité et selon les pédagogies des unités elles-mêmes. A ce jour, aucune police allemande ne dispose de villes spécialement construites pour l'entraînement au maintien de l'ordre, ce qui n'est pas sans conséquences notables sur la conception de ces opérations, comme nous le montrerons plus bas. L'entraînement spécifique au maintien de l'ordre a lieu dans des centres de formation de la police où les enseignements sont généralement dispensés dans des salles de classe. Ces centres sont temporairement transformés en vue d'accueillir ces entraînements en extérieur : ce sont les routes qui servent alors de théâtre d'opération. Celui que nous avons observé est un cycle de formation destiné aux commandant.e.s d'unités de maintien de l'ordre (Gruppenführer*innen), qui répercutent ensuite les fruits de cet entraînement, de manière décentralisée, dans leurs unités respectives.

Concernant la méthode employée, je m'appuie sur une méthodologie empruntée à l'ethnographie (Lofland, 1971; Hammersely, Atkinson 1983), qui permet de saisir à la fois les espaces, les pratiques et les artefacts (Baur, Hering, $2017: 390$ ), mais qui permet également aux perspectives subjectives «d'être reconstituées selon le sens que les interlocuteurs leur ont donné " (Hitzler, 2006: 50). Plus précisément, nous nous référons à l'ethnographie institutionnelle, proposée par Smith (1987). Cette méthode est employée en vue de lier des phénomènes sociaux locaux à leurs conditions de production institutionnelles ou politico-juridiques. L'apport de cette méthode consiste à prendre en compte dans un même mouvement les observations ethnographiques menées à l'échelle micro et les contextes plus larges (voir aussi Campbell, Manicom, 1995 :11). Dans le cadre de cette méthode, aucune différence n'est faite entre les perspectives micro et les perspectives macro (Smith 1987 : 56). Pour la problématique qui est la nôtre, une telle méthode s'avère fructueuse, dans la mesure où aucune simulation n'est concevable sans référence externe - au « réel » - et donc à un contexte plus large. Différents éléments entrent en ligne de compte pour influencer les modalités du maintien de l'ordre, par exemple les structures institutionnelles, les influences politiques, des présupposés juridiques ou plus largement des ambiances sociales (à ce propos voir Joyce, Wayne, 2014). 


\section{L'aménagement spatial de la ville artificielle : constitution et reproduction de quartiers « à risque »}

14 Comment ces espaces urbains sont-ils concrètement constitués et à quels usages sontils destinés? Les résultats de l'analyse de nos observations, des entretiens et de l'évaluation des documents de police, que nous présenterons ci-dessous, montrent généralement ce qui suit: ces villes artificielles sont en premier lieu le fruit des imaginaires policiers liés à l'ordre public et plus particulièrement au maintien de l'ordre public. Les policiers-formateurs à qui nous avons parlé ont expliqué qu'ils font part de leurs besoins, de leurs représentations et des configurations de l'espace nécessaires aux entrainements aux bureaux de conception et d'architecte ou encore de BTP durant les phases préparatoires des travaux. Dans les cas étudiés, ces villes sont généralement décrites comme typiquement européennes. Dans le cas français, le centre de formation propose également la reconstitution d'une "zone rurale ». Dans tous les centres d'entrainement examinés, il revient à l'administration de la police de doter ces installations de diverses parures » urbaines » : panneaux de signalisation, pancartes de commerces, etc. Un membre de la police britannique note en effet :

L'exigence des administrations policières dans tous les pays consiste à construire et aménager des espaces "aussi réalistes que possibles" (Formateur Williams, Angleterre, Metropolitan Police, IEE6, 21.06.2018).

Nos interlocuteurs policiers nous font souvent part du fait qu'il s'agit de reproduire des installations urbaines «normales». Une attention plus resserrée fait en réalité ressortir des différences notables avec des sections urbaines ordinaires. Les villes ainsi reconstituées ont tendance à dépeindre des quartiers populaire perçus comme dangereux, où les problèmes sociaux semblent avoir un impact profond sur la vie quotidienne. D'autre part, les espaces sont représentés comme étant eux-mêmes en danger ${ }^{7}$. Certaines actions sont perçues comme problématiques parce qu'elles menacent l'espace dans lequel elles se produisent, souvent les centres-villes, en y introduisant le risque d'actions violentes, de dommages aux biens, et de désordre en général. Les polices reproduisent ainsi dans l'espace l'environnement qu'elles estiment lié à des groupes de populations ou des groupes protestataires sur lesquels elles concentrent déjà leur attention dans leur travail quotidien. En France par exemple, une partie du Centre de formation reproduit un centre-ville pauvre, où, selon la simulation, il peut y avoir une zone vulnérable (un espace pensé comme étant en danger), une autre partie du Centre est présentée comme une ville d'outre-mer française ou bien une cité de banlieue, une troisième partie enfin une ZAD (Zone à défendre) de milieu rural. Ces lieux sont décrits par les policiers comme des lieux où le maintien de l'ordre s'inscrit dans un contexte criminel habituel. Un des formateurs français explique, à propos de la section reproduisant la banlieue et la ville d'outre-mer :

" On a un pôle 2 qui est plus dédié, je dirais qui a une double destination, à savoir tout ce qui est franchissement [...]. Donc ça sera mis en action aussi, tu le verras lors $\mathrm{du}$ thème 'maintien de l'ordre outre-mer' [...], une problématique d'interpellation d'individus donc qui ont commis des meurtres etc. et en même temps une thématique d'ordre public » (Interview IFE2, France, formateur W. Boulanger, 14.11.2018).

En Angleterre et en Irlande du Nord, c'est un quartier défavorisé, plus précisément un centre-ville pauvre, qui est représenté. En Allemagne, les formations ont lieu dans des espaces qui ne sont pas spécifiquement conçus pour les entrainements en situation et 
qui par conséquent n'ont donc pas de vocation particulière. Ces espaces de formation ressemblent néanmoins à des quartiers périphériques. Ainsi toutes les installations fictives, à l'exception de celles présentes en Allemagne, ancrent l'espace urbain dans des contextes de pauvreté ou de marginalités sociales, où des attitudes collectives de défiance à l'égard de l'État voire de présence de groupes contestataires sont supposées. Elles mettent aussi en scène les priorités ou enjeux du moment, comme la ZAD du centre de St Astier, qui est construite sur le modèle de la ZAD de Notre-Dame-desLandes (notes de terrain, 14.11.2018).

Cette référence à des contextes défavorisés peut être illustrée par l'exemple des forces de police anglaises dans la région de Londres : la «ville d'entrainement» des polices anglaises, qui reproduit une section urbaine dont on ne sait toutefois pas s'il s'agit d'une grosse ville ou d'une petite ville, consiste en une série de rues rectilignes, qui se croisent à angles droits. Trois séries de trois rues forment un quartier dont des maisons, des façades ou des murs occupent le paysage. A côté de cette section construite se trouve un petit espace vert, ceint par une rue et fermé sur un côté par une rangée de maisons.

Dans la ville fictive des polices de Londres se présente ainsi un mélange typique d'infrastructures urbaines avec des entreprises et des habitations: on y trouve entre autres un pub, une pizzeria, une station de métro (au rez-de-chaussée d'une habitation et ainsi un peu en retrait de la rue) flanquée de deux wagons, une boîte aux lettres et des habitations. Un poste de police, avec cellules de garde-à-vue, se trouve en bordure de l'espace vert. Des panneaux indiquent les noms des commerces: la pizzeria est appelée Pizzaland, ce qui suggère une association avec une chaîne de distribution de pizzas, un service de livraison ou en tout cas un établissement bas de gamme. A côté de ce restaurant bon marché, se trouvent également un teinturier («Cleaners»), une agence de voyage («Travelworld») et un magasin de vêtements (« Top Gear »). La ville ainsi reconstruite est clairement un centre-ville, centrée autour d'une « Main Street »: tous les commerces se trouvent sur la rue principale, qui est également le centre géographique de la ville.

Selon un formateur, nous avons là une «ville anglaise typique », typique du point de vue des interventions policières de la Metropolitan Police, de la London City Police et la British Transport Police. Aucun type de quartier en particulier n'aurait servi de modèle :

« Il n'est pas modelé sur un lieu géographique ou un environnement particulier ou quoi que ce soit, il fournit simplement des routes de différentes largeurs, des ruelles, des carrefours, des jonctions en $\mathrm{T}$, des routes qui ont des bâtiments de deux étages, des routes qui n'ont pas de bâtiments du tout.... Disons qu'il fournit un large spectre » (Interview IEE3, Angleterre, Constable Potter, 19.06.2018).

Si l'on regarde toutefois attentivement la ville ainsi reconstruite, on remarque qu'elle ne présente pas le découpage urbain propre à n'importe quelle partie ville anglaise. Avec ses coursives traversantes, ses passages entre les différents blocs de maisons et la structure particulièrement étroite et enchevêtrée de ses cours intérieures, les habitations suggèrent une ville ou un quartier peu aisé. Certes, la forme ainsi représentée des blocs à angle droit avec cours intérieures dotés d'espaces verts et les commerces donnant sur la rue principale incarnent « la forme normale des habitations tant bourgeoises qu'ouvrières » (Sonne 2017 : 110). Cependant, l'étroitesse particulière des structures architecturales qui ressort de l'écart entre les bâtiments, de la taille des cours, des voies piétonnes entre les blocs qui se font face ou de la maigreur des espaces 
verts suggèrent une représentation typique d'habitations propres au pouvoir d'achat de personnes de la petite classe moyenne et des milieux sociaux les plus bas; tout suggère ici un quartier ouvrier ou de petite condition.

C'est pourquoi, que ce soit en France, en Angleterre ou en Irlande du Nord, ce sont des configurations spatiales bien spécifiques qui sont conçues pour les entrainements policiers. Si l'on se réfère à la sociologie de l'espace et de l'architecture, on peut affirmer que dans ces villes artificielles, la matérialité des productions architectoniques et des aménagements spatiaux crée les usages ; ceux-ci correspondent à des fonctions spécifiques et anticipent même ces fonctions (voir dans le même sens: Foster, 2004). Dans un tel contexte, les espaces construits pour la police font naitre des formes d'action sociale particulières : ils imposent " où on est et ce qu'on a à faire " (Schroer, 2013 : 20). Ces "villes» peuvent ainsi être considérées comme représentant des "ordres sociaux» spécifiques, l'espace étant préconstruit par le biais de données matérielles et de représentations, qui en tant que telles incitent à certaines pratiques spatiales et rendent plus difficiles voire interdisent d'autres pratiques (voir plus bas). Ainsi, non seulement "l'architecture organise la condition sociale de la police" (Lambert, $2016: 8$ ) mais elle organise également l'espace construit à des fins policières. En dépit d'une aspiration des policiers à «l'authenticité » des espaces urbains, le maintien de l'ordre est lié à des segments bien déterminés de la population par l'aménagement des espaces d'entrainements. Ceux-ci reflètent les représentations socioculturelles attachées aux populations vues comme adverses et révèlent par leur agencement même les dangers potentiels qu'ils abritent.

Si les espaces aménagés en France et en Angleterre sont censés évoquer des conflits permanents, les espaces d'entrainement de Basse-Saxe font songer à un quartier de la périphérie urbaine calme et apaisée. Le centre où se déroulent les formations dispose de locaux relativement modernes, puisqu'il a été équipé dans les années 1980 pour assurer le maintien de l'ordre lors des manifestations contre le transport de déchets nucléaires par le train Castor vers le centre de stockage provisoire de Gorleben, qui se sont déroulées à grande échelle jusqu'en 2000. Le centre du complexe est formé par une pelouse ovale autour de laquelle est regroupée l'administration du centre de conférence sous la forme d'un bureau de conférence et d'une cantine, ainsi que de quelques dortoirs et bâtiments de formation; ceux-ci sont reliés par un chemin piétonnier faisant le tour de la pelouse. La partie décrite forme un côté de la zone rectangulaire oblongue. De l'autre côté, relié au milieu par un rond-point, se trouve une zone de taille similaire caractérisée par un ancien point de rassemblement des personnes interpellées pendant les manifestations contre le Castor, quelques bâtiments de dortoirs et de conférences, un grand parking et une prairie qui s'étend sur tout le côté latéral à l'extrémité. Sur le côté, au milieu de ces deux parties, se trouvent l'entrée de la propriété et une station-service pour les véhicules de police. Aucun des bâtiments n'a plus de deux étages. D'un point de vue structurel, on peut parler ici d'un modèle de base presque anti-urbain, qui se caractérise par une faible densité de peuplement et une forte insistance simultanée sur les voies de circulation pour le trafic individuel. Il évoque des associations avec une construction et une planification urbaine moderne qui rompt avec les structures urbaines historiques. De telles structures urbaines sont apparues en Europe principalement dans les années 1960 à 1990 sous la forme de villes bâties à la périphérie des agglomérations. De plus en plus, des "villes intermédiaires" sont construites, qui n'ont pas nécessairement un centre clairement identifiable. L'idéal 
de vie des cités pavillonnaires renvoie, comme d'ailleurs dans les «villes fonctionnelles" de Le Corbusier, à la ville comme outil de pacification et comme alternative aux bouleversements sociaux (Howard, 1907). Ces formes urbaines étaient pensées comme libérées de l'histoire, du chaos, de la misère et des émeutes. Transposé aux installations policières, cela signifie que les simulations de maintien de l'ordre prennent place dans un environnement urbain caractérisé par l'absence de conflit.

\section{L'organisation pratique de la ville artificielle comme " espace d'expérience »}

23 L'objectif de ces espaces d'entrainement est de parvenir à la présentation la plus « authentique » possible (pour reprendre un qualificatif récurrent dans les entretiens réalisés avec des policiers), non seulement en ce qui concerne la morphologie urbaine, mais aussi en ce qui concerne les pratiques des policiers dans ces espaces. Les évènements mis en scène doivent refléter la complexité des épisodes contestataires réels, et leur impact sur les policiers engagés dans le maintien de l'ordre. L'enjeu est pour les instructeurs de générer dans ces espaces urbains des situations dans lesquelles les policiers sont amenés le plus près possible de leurs limites personnelles. Les observations montrent qu'au cours de ces entrainements, les policiers sont confrontés principalement à un foisonnement d'événements, au désordre, à l'imprévisible, au bouleversement mais aussi bien sûr au danger. Appuyées sur des éléments spatiaux qui suggèrent en eux-mêmes des dangers et engendrent stress ou peur chez les agents en formation, les situations produites visent à les former à agir de manière prévisible et contrôlée en toutes circonstances (voir sur les entraînements policiers en général: Chappell, Lanza-Kaduce, $2010: 3$ ). La raison doit prendre le pas sur l'émotion. Voici comment le formateur T. Brown décrit le processus :

«Certains protestataires se livrent à des désordres de nature agressive, mais d'une durée très courte, donc nous mettons les policiers sous pression, à la fois mentalement et physiquement » (Interview IEE1, Angleterre, 14.06.2018).

On peut en conclure qu'en travaillant sur le contrôle des émotions négatives - stress, peur, etc. - on cherche à contenir une tendance à l'action incontrôlée, afin que les pratiques des policiers ne redoublent pas «la dynamique émotionnelle des masses humaines " (Collins, $2015: 32$ ) - une problématique à laquelle les forces de l'ordre se disent en permanence confrontées. Au cours de nos séjours sur le terrain, il nous a été fréquemment répété que la sur-réaction du côté des policiers est souvent constatée. Des policiers oublient leurs fondamentaux dans le feu de l'action et sur-réagissent, oubliant qu'ils ont en face d'eux des collègues et qu'ils sont en situation de simulation.

Selon les formateurs, toutes les situations qui peuvent survenir au cours d'interventions réelles doivent avoir été autant que possible éprouvées à l'entraînement. Les policiers usent pour rendre compte de cela du concept "d'expérience ", de la nécessité de " cumuler de l'expérience », ce que l'espace urbain et son utilisation théâtrale sont censés rendre possible. Des émotions sont précisément suscitées, mises en action, afin d'en rendre le contrôle possible. Le formateur A. Dupont du centre national de la gendarmerie formule cette approche en ces termes:

«Il faut que l'entrainement soit difficile, réaliste pour que demain, au moment de

l'engagement opérationnel, il n'y ait pas trop de différence. Que le gendarme n'ait pas l'impression qu'il va être confronté à quelque chose qu'il n'arrivera pas à 
surmonter parce qu'il ne l'aura jamais connu avant. Donc c'est vraiment l'idée, voilà : être le plus réaliste possible par les moyens de simulation, par le fait de se tenir informé de ce qui se passe sur les vraies opérations pour ensuite adapter ce qu'on voit dans les vraies opérations sur les exercices » (IFE7, France, 20.11.2018).

Il ajoute :

"Comme vous le savez ici on a... On a une devise ou un leitmotiv qui est: le gendarme agit comme il s'entraîne, il doit s'entrainer comme il veut agir » (ibid.).

Cette phrase figure d'ailleurs à l'entrée du centre sur un panneau d'environ 5 mètres de long.

Tout ceci signifie que les polices s'entraînent au cours des simulations à des techniques qui ne peuvent être isolées les unes des autres, mais qui au contraire se rapportent à un enchainement complexe. La complexité des évènements mis en scène est accrue au moyen d'une représentation sensible de la vie sociale. Les formateurs s'attachent ainsi à proposer des entraînements dits " dynamiques », caractérisés par une augmentation du degré de difficulté, dans lequel les techniques et tactiques policières enseignées sont intégrées dans la complexité d'un contexte social spatialement constitué (voir par comparaison les entraînements militaires in Kretschmann, 2016).

9 Dans cette perspective, l'équipement de l'espace bâti fait l'objet d'une attention particulière, notamment pour y introduire «de la vie ", comme le disent souvent les acteurs concernés. Ainsi, les lieux servant à l'entraînement sont équipés des artefacts de cette « vie ", comme des pavés (selon les lieux en bois, en plastique ou sous forme de balles de tennis) ou des voitures brûlées. Dans cet espace aménagé, on tente une reproduction de ce qui est vu comme la culture protestataire et des protestataires euxmêmes. Ainsi les policiers jouant le rôle des manifestants brandissent des pancartes ou des banderoles, mais ils viennent aussi habillés de manière "typique ", c'est-à-dire le plus souvent entièrement vêtus de noir, afin d'incarner par exemple des black blocks. Cette mise en scène montre que l'essentiel des forces manifestantes représentées sont des forces de gauche et, dans les simulations, la police assimile toute protestation de gauche (de l'extrême-gauche à la gauche libérale) au Black Block. Des policiers expliquent également que pour incarner les manifestants, ils se sont préparés par quelques recherches sur Internet. "Ils doivent entonner des slogans et déclamer des discours ", " il est de toute première importance que nous soyons au plus près de la réalité ", nous ont dit les formateurs sur chacun des terrains étudiés.

Même si en Allemagne les entraînements ne se déroulent pas dans des villes spécialement reconstituées, les tentatives de faire vivre les espaces y sont également à l'œuvre. Là-bas, une feuille A4 indique un foyer de demandeurs d'asile, et d'autres affichettes identifient un bar et la résidence d'un particulier. Mais dans ces paysages aussi les manifestants portent des vêtements noirs et des banderoles. Sont ainsi créés au cours de l'entraînement un savoir corporel et des sentiments chez les policiers qui seraient mis à l'épreuve de manière similaire dans une intervention réelle. C'est la raison pour laquelle nous pouvons qualifier ces villes artificielles - suivant en cela l'autodescription des policiers - d'espaces expérientiels. Ainsi, ces espaces sont conçus pour mettre en scène certaines expériences, lesquelles entrent à leur tour en résonance avec la manière donc ces lieux sont conçus. Les "expériences » ainsi mises en scène font en effet, dans l'utilisation pratique des lieux, plutôt référence à des mouvements de gauche qui sont, pour les policiers, les plus enclins à la violence. 


\section{La constitution de " géographies assaillantes » dans la ville artificielle}

31 Les simulations sont structurées de manière à proposer aux policiers des situations d'intervention dans lesquelles ils s'exercent à mobiliser des éléments particuliers propre à la pratique du maintien de l'ordre. Le comportement de la foule dans l'espace urbain est en ce sens conçu comme un événement qui appelle une réaction tactique et technique spécifique de la part des forces de police. Pratiques et éléments spatiaux se confondent ici pour produire des représentations particulières de situations problématiques. Un extrait d'entretien avec le formateur A. Klaussen de la police de Basse-Saxe illustre cette idée de manière exemplaire :

«Quelle que soit la situation, il s'agit d'un comportement spécifique. Je veux en partie provoquer, pour rendre ce qui se produit de la manière la plus nette auprès des concernés » (IDE3, Allemagne, 04.09.2018).

On entraîne ainsi l'unité à se positionner, à se mouvoir et à se comporter à l'égard de la foule. En même temps, on enseigne aux policiers comment garantir leur propre sécurité et celle de la foule :

« Et tout ça en gardant à l'esprit qu'évidemment nous rentrerons à la maison en bon état et que nous ne voulons aucun blessé. Dans cet esprit, l'évaluation de la situation joue un rôle décisif : pas juste rentrer dans le tas, juste parce que la police peut le faire, non, mon dieu, jamais! Le retrait est toujours une option disponible même si bien sûr personne ne veut se donner à voir comme l'élément faible " (IDE3, Allemagne, 04.09.2018).

Pour élever le niveau de difficulté de l'entrainement, la ville policière, que ce soit en France, en Angleterre ou en Irlande du Nord, va au-delà d'une situation de manifestation; on ne peut pas vraiment parler ici "d'authenticité ", ni de cette aspiration à « la réalité » évoquées par les policiers. Nos observations montrent que sur ces lieux, après quelques minutes, les affrontements tournent plutôt à «la guerre ", «Armageddon» ou au «chaos", pour reprendre des mots employés de nombreuses fois par les policiers, les uns avec malice, les autres avec l'envie manifeste d'en découdre. Cela se traduit concrètement par le fait que, après une courte phase préliminaire de manifestation pacifique, ce sont en fait des scénarios proches de situations de guerre civile qui sont joués. Tous les événements protestataires pour lesquels on s'entraîne dans des villes artificielles sont ceux que les policiers définissent comme des événements à hauts risques. Ainsi, nous avons pu observer qu'environ 40 cocktails Molotov sont jetés à chaque entraînement des polices anglaises, et que les pierres sont également massivement employées, après avoir été acheminées dans des caddies par les manifestants. Si les pierres sont en bois, les cocktails Molotov, eux, sont réels : la quantité de liquide inflammable utilisée est limitée et les bouteilles sont faites d'un verre fin qui donne un effet un peu plus intense que celui d'un cocktail Molotov «standard ». La foule est le plus souvent représentée de manière indifférenciée comme une masse encline à employer la violence et propre à engendrer du danger: les policiers doivent d'emblée compter avec le fait que les manifestants vont employer la violence. Ainsi, chaque événement protestataire simulé, qui a presque toujours l'allure d'un rassemblement de gauche, se déroule de manière violente, dangereuse, à la frontière de la guerre civile. Pour le dire autrement: le maintien de l'ordre lors des manifestations est présenté comme équivalent au maintien de l'ordre en guerre civile. En conséquence les forces de police emploient toujours le niveau maximal de force dans 
leur réponse. Un camion lanceur d'eau est toujours présent lors des grandes opérations de simulation en Irlande du Nord et de son côté la gendarmerie française emploie de 40 à 60 grenades de différents types par entraînement.

La conséquence de ces dispositifs est que les simulations en France, en Angleterre et en Irlande du Nord ne représentent pas des manifestations ordinaires, car la grande majorité des manifestations se déroulent de manière pacifique, dans le cadre prévu par la loi (Meyer, Tarrow 1998 ; Fillieule, Jobard 1998). Plus précisément, ces entraînements ont vocation à préparer à toutes les situations, c'est-à-dire en réalité à la pire des situations, aux scénarios apocalyptiques d'affrontement violents qui finissent toujours par arriver dans le déroulé des entrainements construits sur un modèle d'escalade continue. Que de tels niveaux d'escalade soient rarement constatés sur le terrain (les policiers anglais indiquaient par exemple que les deux derniers cocktail Molotov qu'ils ont pu voir dataient des émeutes de Londres en 2011), est selon les policiers d'une importance secondaire. Et même en France, où les niveaux d'intensité des manifestations sont beaucoup plus élevés que dans les autres pays étudiés, l'entraînement est de toute façon fondé sur une logique d'inévitable escalade. " Entraînement dur, guerre facile ", comme nous l'ont dit maintes fois les gendarmes français.

Dans la ville artificielle française, les entraînements ont souvent pour point de départ des pièges tendus à la police dans les banlieues. Ces scénarios catastrophes se traduisent de manière concrète dans l'architecture des villes : les maisons sont érigées de manière à pouvoir être utilisées pour jeter des objets sur les policiers. En outre, une impasse a été créée pour rendre plus difficile la retraite de la police, car elle ne peut s'effectuer que d'un seul côté. Dans le centre d'entraînement des polices de Londres, toute une série de connexions ont été établies entre les entrées d'immeubles, avec des couloirs et des coursives agencés de manière à permettre le jet de projectiles et de la fuite des manifestants. L'intensité des affrontements est également inscrite dans l'espace : même lorsque la ville est calme, des marques d'incendie sur le bitume ou sur les immeubles, des dégradations sur le bâti, ou encore des fragments de pierres et des éclats de verre des cocktails Molotov montrent la violence des simulations. Le centre allemand fait figure d'exception: la violence n'y est pas considérée comme une étape inévitable des manifestations - lorsqu'elle se produit durant les entrainements, elle est beaucoup plus limitée -, et en conséquence aucun conflit n'est inscrit dans l'architecture urbaine. Les espaces policiers en France, en Angleterre et en Irlande du Nord sont ainsi codés comme des espaces dangereux. On peut les considérer comme faisant partie de ce que John P. Crank appelle les "géographies assaillantes»: des "zones publiques et privées qui présentent des signes que des agents expérimentés reconnaissent comme potentiellement dangereux » (Crank, 2004:161).

Pour décrire la logique du scénario catastrophe, la recherche en criminologie, qui jusqu'à présent l'avait réservée au domaine du terrorisme, est utile : cette logique est organisée autour d'une perception particulière du futur, où le pire est toujours certain (Aradau 2010; Horn 2007). La survenue de la catastrophe correspond à une défaite totale de l'autorité étatique, en l'occurrence avec au moins une autorité étatique sapée et une police totalement délégitimée. Ces évènements, indépendamment de leur plausibilité, mettent en scène des futurs systématiquement apocalyptiques qui imposent une conscience exacerbée du danger et du risque potentiel (Kretschmann, 2012). En référence à Engell et al. (2009), on peut parler ici d'un « sens du danger » qui 
s'établit à travers le scénario catastrophe. Au regard de ces notions, on peut affirmer que la plupart des polices étudiées représentent des villes confrontées en permanence à des situations de menace et de crise aigües.

La police de Basse-Saxe emprunte, seule, une autre voie, comme le montrent nos recherches empiriques. Sa direction préfère en effet mettre l'accent sur les événements de moyenne intensité. Un formateur précise :

«Il est très rare que le pire des cas arrive, mais de temps en temps, un tel exercice est joué afin de montrer que même si vous êtes en opération, les choses pourraient très mal tourner » (IDE8, Allemagne, Instructeur G. Woltmann).

La plupart du temps les entrainements consistent en des simulations réalisées en petits groupes, qui mettent en scène des situations de la vie quotidienne. En quelques occasions seulement des scènes de type guerre civile comparables à celles des autres pays sont déployées. Mais dans les entraînements les plus communs, une grande valeur est accordée à la qualité de l'interprétation et du «jeu » des acteurs : les scénarios se concentrent moins sur des situations d'escalade rapides que sur des situations à problématiques de moindre intensité, par exemple avec des manifestants qui entreprennent de contourner une voie barrée ou qui commencent un sit-in. Ces scénarios cherchent à éprouver les habitudes d'intervention et de réaction des forces de l'ordre dans des contextes plus pacifiques. La police de Basse-Saxe colle ainsi davantage au vraisemblable qu'à une vision apocalyptique des manifestations. En un certain sens, l'espace est moins utilisé en Allemagne que dans les autres pays: l'aménagement $d u$ site d'entrainement ne leur permet en réalité que ce type d'entraînement de basse intensité. Les forces de police ne disposent pas d'espaces urbains artificiels spécifiques pour leurs simulations: les espaces utilisés sont également des espaces de travail, de dortoirs, de salles de classe, de cantines, etc., de telle sorte que les situations d'escalade ne peuvent y être travaillées que de manière limitée. Dès lors, l'espace de formation de la police de Basse-Saxe est bien moins souvent utilisé pour représenter les géographies assaillantes que chez les autres polices européennes: l'espace ne porte pas la marque de groupes sociaux spécifiques, par exemple des quartiers périphériques, ni la marque visible d'activités criminelles ou délinquantes. En conséquence, quelques simulations se déroulent si paisiblement qu'aucune intervention du groupe jouant la police n'est nécessaire.

\section{Conclusion : des espaces « autres »}

Nous avons établi que les espaces des villes artificielles étaient «produites » (au sens littéral du terme) criminogènes. Leur caractère imaginaire rejoint les observations de Michel Foucault relatives à sa conception des lieux hétérotopiques comme espaces "créés». Ils sont "un autre espace, un autre espace réel, aussi parfait, aussi méticuleux, aussi bien arrangé que le nôtre est désordonné, mal agencé et brouillon » (1984: 759). Nous avons montré dans l'analyse ci-dessus que les centres d'entraînement sont des lieux dans lesquels la police s'exerce à ce qui doit se produire « en vrai », pour reprendre l'expression des policiers, dans des conditions contrôlées et dans un cadre dépourvu de risques. A cette fin, la police met en scène des espaces manifestants, des manifestations et des manifestant.es fictifs, produits à partir de leurs représentations et perceptions de ce qu'est la « réalité »et qui appellent en retour des réponses spécifiquement policières. Ici, les rationalités policières pré-existent aux 
espaces, mais les espaces eux-mêmes exercent leurs effets spécifiques - et prévus - sur les rationalités policières. Contrairement au vœu des polices consistant à prévoir des représentations les plus réalistes possibles, on observe en de maintes occasions de fortes divergences, volontaires tant qu'involontaires, par rapport à la réalité. La logique et les possibles effets de ces divergences doivent être interrogés au regard de la question formulée au début de cet article sur la contribution de la police à la formation de l'ordre politique.

La sociologie considère que les espaces clos disposent de leurs propres règles et de leurs propres systèmes, et, par conséquent qu'ils construisent leur propre réalité (Berger, 1998 : 8). Durant les entraînements, qui pratiquent l'atteinte aux règles selon des règles spécifiques, la question des réalités construites se pose au regard de ce qui est représenté et de ce qui est déduit de la représentation. Ainsi, il est établi que les événements protestataires pour lesquels on s'entraîne dans les villes artificielles sont ceux définis par la police comme des événements à hauts risques. C'est avec ces événements que les policiers (à l'exception des policiers allemands) bâtissent leur expérience, expérience selon laquelle tout événement protestataire est violent et dangereux et en permanence à la frontière de la guerre civile. En conséquence, laisser venir l'événement à soi n'est tout simplement pas une option: ce scénario apocalyptique formule une urgence et pose un impératif d'action. Même les actes conformes au droit de manifester sont vus comme porteurs de dangers et d'infractions. Par exemple, en France les policiers insistent sur le fait que même un manifestant encore pacifique, comme on en voit au cours de la simulation, peut devenir violent l'instant suivant.

41 Tout ceci marque le regard que les policiers peuvent porter sur les manifestations et les manifestant.es, et consolide les stéréotypes déjà en circulation. Ces interventions simulées formatent également la perception de ce qu'est une réaction policière appropriée: puisque chaque participant aux entrainements sait qu'un maintien de l'ordre dur est attendu en toutes circonstances puisque ce sont systématiquement des situations-catastrophes qui sont mises en scène. Les compétences attendues en contexte de manifestation pacifique sont tout simplement supposées acquises - il s'agit d'interventions faciles, qui n'appellent pas d'entrainements particuliers; en conséquence, le temps dévolu à ces entraînements est réduit à la portion congrue, sauf en Allemagne. Les policiers sont sélectionnés aussi à partir de leurs compétences sociales afin qu'ils puissent faire face à de telles situations de manière indépendante. Toutefois, ce point de vue est contrebalancé par les observations d'un public largement critique, qui considère précisément la dynamique de la désescalade comme sujette à l'erreur'. Les simulations de forces de police ont donc des conséquences sur la participation politique et soulèvent à cet égard des questions de politique démocratique.

42 La particularité de cette forme d'entraînement est que les aspects de la vie quotidienne, aussi bien sociaux que culturels, sont intégrés aux situations d'entraînement et sont l'objet d'anticipations. C'est une différence majeure entre les formations simulées et les formations sur le terrain : la prise en compte des contextes sociaux dans l'entrainement signifie que ces derniers acquièrent une dimension réelle aux yeux des forces de l'ordre. C'est précisément ce que visent les efforts d'authenticité entrepris par les entraîneurs.ses au cours des simulations : en dépit de toutes les différences entre les scénarios déroulés et les manifestations traditionnelles ou les émeutes, les simulations 
transmettent aux policiers en intervention une impression d'événement réel. Les policiers ne sont pas seulement entraînés aux techniques et aux tactiques de maintien de l'ordre. Afin qu'ils anticipent les actions des protestataires qu'il s'agit de contrôler, les modus operandi de ces derniers leur sont enseignés avec le plus de détails possibles. Bien sûr, les polices ont toujours fait reposer leurs stratégies sur la connaissance et le renseignement, afin de parer à toute contingence : l'histoire des registres, des fichiers, des photographies, etc., les connaissances accumulées sur les milieux criminels le montrent suffisamment (Becker, 2005). Tout ceci est désormais condensé dans les villes artificielles, dans une large mesure, spatialement, et par conséquent également de manière corporelle et sensible. Cet état de fait peut marquer les policiers et façonner un maintien de l'ordre imprégné de considérations particulièrement fortes quant à leur perception de la société.

Cette dynamique de simulations peut être vue comme un acte de formation d'une réalité et d'un ordre politique au moyen de la création «d'espaces autres », au sens de Michel Foucault, des lieux qui s'offrent au contrôle et à l'ordre, ne serait-ce que de manière expérimentale. Les modalités d'entrainement de la police reviennent ainsi à produire la réalité par la simulation : la réalité des entrainements, mais aussi celle des interventions de maintien de l'ordre.

Ces lieux contribuent ainsi à la production d'un ordre politique, étant partie prenante du processus politique contemporain de sécurisation (Buzan, Waever, Wilde, 1998) de la protestation, qui s'inscrit plus généralement dans une tendance à la systématisation d'une police préventive, pilier de la politique de lutte contre la criminalité, qui envisage d'anticiper le danger et d'empêcher qu'il ne se produise. La criminalité est, dans ce contexte, reconceptualisée comme risque, objet de prévision statistique (SchmidtSemisch 2002), qu'il s'agit d'empêcher avant son occurrence, par une action appropriée. L'enjeu est alors moins de poursuivre des délits que de rechercher à re-stabiliser des désordres partiels, par des mesures de lutte contre la délinquance de plus en plus appuyées sur le calcul des risques qui sont associés à des espaces, des situations et des populations (Lianos, Douglas, 2000). Dans cette dynamique, la perception des espaces par la police contribue à peser sur les représentations sociales partagées des espaces urbains, devenus lieux d'insécurité (Häfele, 2011).

La représentation et le traitement des manifestations sont également marqués par ces pratiques et par ces perceptions policières de l'espace. En effet, la prévention se confond dans ce cas avec la notion d'empêchement du risque : si les manifestations, par définition des événements qui se produisent dans l'espace public, font l'objet de régulation par la police (à la fois en vue d'assurer la sécurité des manifestants et de garantir l'ordre public), celle-ci les considère et les traite de plus en en les classant par degré de risque et en intensifiant une logique du praevenire. L'anticipation du risque s'émancipe ainsi du concept de menace imminente pour lui préférer celui de danger diffus, légitimant une intervention beaucoup plus précoce et problématique au regard de la protection des droits fondamentaux. L'issue de ce processus est l'encagement de la manifestation dans des zones données. La Russie et le Canada offrent des exemples éclatants de cette conception étatique de la manifestation comme perturbation: pendant les Jeux olympiques en Russie et au Canada, les espaces concédés à la manifestation étaient systématiquement des zones fermées, situées loin des cibles de la manifestation. En Russie, le nombre de participants était limité et l'identité des manifestants contrôlée (Coaffee, 2016). 
Dans le même temps, les doctrines visant la désescalade, l'évitement de la confrontation et le dialogue sont dominantes. Mais les manifestations, qui sur la base des renseignements sont identifiées comme à "hauts risques", sont de plus en plus considérées comme d'autres événements relevant de mesures pénales (Joyce, Wain, 2014 : 274, Fillieule, et al., 2016). Ce type d'événements n'est plus vu comme relevant du domaine de l'expression civique, mais comme du domaine de l'occupation illégale, selon une logique d'incapacitation sélective: les possibilités de dialogue sont dès le départ fortement circonscrites et les seuils autorisant la confrontation directe et l'escalade abaissés. Les pratiques illibérales paraissent relever du domaine de l'opportunité (Kretschmann, 2014). Dans un tel contexte, se pose la distinction conceptualisée par Donatella della Porta et Herbert Reiter qui oppose la police « du citoyen » de la police «du gouvernement» (2006: 113ff). Celles-ci accompagnent et favorisent une extension de la tactique d'occupation policière avec les manifestations. On a notamment pu observer une radicalisation et une extension des mesures appliquées aux manifestations estampillées comme à hauts risques dans le sillage de la crise économique et financière de 2008 et du tournant autoritaire d'un certain nombre de sociétés européennes (Kretschmann, 2014 ; Ullrich, 2012). Il y a un accroissement de la densité de la réglementation, du contrôle et de la coopération en matière d'ordre public, mais aussi une inclination à considérer les manifestations comme des activités criminogènes, dangereuses et perturbatrices, devant être spatialement refoulées (Petzold, Pichl, 2012).

Les entraînements en simulation dans les villes artificielles encouragent cette tendance de manière prononcée : parce qu'ils présentent des situations exacerbées de danger et d'escalade, ils encouragent la mise en scène de manifestations comme événements appelant un contrôle de plus en plus resserré. De ce point de vue, les différences structurelles entre les types de police (Jobard, de Maillard, 2015: 47ff.), les représentations qu'elles ont d'elles-mêmes (Bowling, Sheptycki, 2012), les doctrines de maintien de l'ordre (della Porta, Fillieule, 2006) et les orientations des formations (Pagon, et al., 1996 ; Jobard, de Maillard, 2015 : 136) comptent finalement peu : même si les simulations laissent paraître des différences notables, la majorité des polices s'exerce à l'emploi d'une force maximale en réaction à une masse indifférenciée, qui ne jurerait, selon elles, que par des situations de guerre civile. Cet aspect n'est pratiquement jamais problématisé par la police. Seuls quelques policiers, dans les entretiens ou au cours des conversations, ont indiqué qu'il est problématique que les centres d'entraînement induisent un perpétuel conditionnement au combat. La police de Basse-Saxe semble néanmoins tracer un chemin différent. Son expérience enseigne que les simulations en entraînement peuvent être employées à d'autres fins, en l'espèce une police orientée par le service rendu aux citoyens. L'espace ici influence clairement - et d'une manière différente par rapport à d'autres pays - la formation et, par conséquent, la réflexion sur les stratégies policières de gestion des manifestations. 


\section{BIBLIOGRAPHIE}

ARADAU C. (2015), « 'Crowded Places Are Everywhere We Go'. Crowds, Emergency, Politics », Theory, Culture, and Society, vol. 32 no. 2, pp. 155-175.

BAUR N., HERING L. (2017), « Die Kombination von ethnografischer Beobachtung und standardisierter Befragung ", Kölner Zeitschrift für Soziologie und Sozialpsychologie, vol. 69 no. 2, pp. 387-414.

BECKER P. (2005), Dem Täter auf der Spur. Eine Geschichte der Kriminalistik, Darmstadt, Primus. BELINA B., WEHRHEIM J. (2011), « 'Gefahrengebiete’ : durch die Abstraktion vom Sozialen zur Reproduktion gesellschaftlicher Strukturen », Soziale Probleme, vol. 23 no. 2, pp. 207-229.

BERGER P. L. (1998), Erlösendes Lachen. Das Komische in der menschlichen Erfahrung, Berlin, de Gruyter.

BERLIÈRE J.-M., LÉVY, R. (2011), Histoire des polices en France. De l'Ancien Régime à nos jours, Paris, Nouveau Monde.

BIALASIEWICZ L., CAMPBELL D., ELDEN S. U.A. (2007), « Performing Security », Political Geography, vol. 26, pp. 405-422.

BOWLING B., SHEPTYCKI J. (2012), Global Policing, Los Angeles u.a., Sage.

BRION F., REA A. (1992), « La construction médiatique et politique des émeutes urbaines », L'année sociale - 1991, pp. 181-203.

BRUNETEAUX P. (1996), Maintenir l'ordre, Paris, Presses de la Fondation nationale des sciences politiques.

BUZAN B., WAEVER O., WILDE J. DE (1998), Security. A New Framework for Analysis, Boulder, Lynne Rienner.

CAMPBELL M., MANICOM A. (Hg.) (1995), Knowledge, experience, and ruling relations. Studies in the Social Organization of Knowledge, Toronto, University of Toronto Press.

CHAPPELL A.T., LANZA-KADUCE L. (2010), « Police Academy Socialization : Understanding the Lessons Learned in a Paramilitary- Bureaucratic Organization », Journal of Contemporary Ethnography, vol. 39, no. 2, pp. 187-214.

COAFFEE J. (2016), « Normalising exceptional public space security. The spatial fix of the Olympic carceral ", in De Backer, M., Melgaço, L., Varna, G., Menichelli, F., Order and Conflict in Public Space, Abingdon, Routledge, pp. 15-36.

CRANK J. P. (2015), Understanding Police Culture, New York, Routledge.

DAVIS M. (2002), Dead Cities, and Other Tales, New York, New Press.

DELLA PORTA D., REITER H. (2006), « Police du gouvernement ou des citoyens ? », in Della Porta, D., Fillieule, O., Police et manifestants, Paris, Presses de Sciences Po, pp. 113-140.

DEVAULT M. L., MCCOY L. (2012), « Investigating Ruling Relations. Dynamics of Interviewing in Institutional Ethnography ", in Gubrium, J. F. et al. (Hg.), The Sage Handbook of Interview Research, 2. ed., Thousand Oaks, pp. 381-397.

ENGELL L., SIEGERT B., VOGL J. (2009) (Hg.), Gefahrensinn, München, Fink. 
FILLIEULE O., JOBARD F. (1998), « The maintenance of order in France. Towards a model of protest policing ", in Della Porta, D., Reiter, H., The Policing of Mass Demonstrations in Contemporary Democracies, Minneapolis, University of Minnesota Press, pp. 70-90.

FILLIEULE O., JOBARD F. (2020), Politiques du désordre. La police des manifestations en France, Paris, Le Seuil.

FILLIEULE O., VIOT P., DESCLOUX G. (2016), « Vers un modèle européen de gestion policière des foules protestataires? ", Revue française de science politique, vol. 66, no. 2, pp. 295-310.

FOSTER N. (2004), « Hightech-Gestaltung - Ästhetik und Nachhaltigkeit prägen die Regeneration der Städte », in Burda, H., Maar, C., Iconic Turn. Die neue Macht der Bilder, Köln, DuMont Buchverlag, pp. 247-259.

FOUCAUlT M. (1984), « Des espaces autres. Hétérotopies », Dits et écrits : 1954-1988, t. IV (1980-1988), Paris, Gallimard, pp. 752-762

GOREAU-PONCEAUD A., PONCEAUD-GOREAU É. (2014), « Le gendarme de Saint-Astier. Les dispositifs spatiaux dédiés au maintien de l'ordre », EchoGéo, no. 28, pp. 1-16.

GRAHAM D. (2011), Cities Under Siege. The New Military Urbanism, London/New York, Verso books.

HÄFELE J. (2011), Die Stadt, das Fremde und die Furcht vor Kriminalität, Wiesbaden, VS.

HAMMERSLEY M., ATKINSON P. (1983), Ethnography : Principles in Practice, London/New York, Tavistock.

HERBERT S. (1997), Policing Space : Territoriality and the Los Angeles Police Department, Minneapolis, University of Minnesota Press.

HITZLER R. (2006), « Ethnografie », in Bohnsack, R. et al., Hauptbegriffe Qualitativer Sozialforschung, UTB, pp. 48-51.

HORN E. (2007), Der geheime Krieg. Verrat, Spionage und moderne Fiktion, Frankfurt am Main, Fischer. HOWARD E. (1907), Gartenstädte in Sicht, Jena, Diederichs.

JOBARD F., DE MAILLARD J. (2015), Sociologie de la police. Organisation politique réforme, Paris, Armand Colin.

JOYCE P., WAIN N. (2014), Public Order Policing, Protest, and Political Violence, Basingstoke/New York, Palgrave.

KALALAHTI J. (2015/16), « How are Simulations used in Security Sector Training in Finnland? », European Police Science and Research Bulletin, no. 13, pp. 70-75

KRETSCHMANN A. (2012) : «Das Wuchern der Gefahr. Einige gesellschaftstheoretische Bemerkungen zur Novelle des Sicherheitspolizeigesetzes 2012 », juridikum, no. 3, pp. 320-333.

KRETSCHMANN A. (2014), « Katalysator Wirtschaftskrise ? Zum Wandel von Protest Policing in Europa », Bürgerrechte \& Polizei, no. 106, pp. 52-62.

KRETSCHMANN A. (2016) : « Krieg und artifizieller Städtebau : Bestandsaufnahme und Problematik », Wissenschaft \& Frieden, no. 2, pp. 11-15.

KRETSCHMANN A., LEGNARO A. (2019), « Die „abstrakte Gefahr“ als Schlüsselbegriff einer Sekuritisierung des Rechts », Zeitschrift für Rechtssoziologie, im Erscheinen.

LIANOS M., DOUGLAS M. (2000), « Dangerization and the End of Deviance. The Institutional Environment », British Journal of Criminology, vol. 40, pp. 61-278.

LAMBERT L. (2016), «Introduction : Architecture is policing », The Funambulist, no. 8, pp. 18-23. 
LOFLAND J. (1971), Analyzing Social Settings : A Guide to Qualitative Observation and Analysis, Belmont : Wadsworth.

MEYER D. S., TARROW S. (1998), « A Movement Society », in Meyer, D.S., Tarrow, S., The Social Movement Society. Contentious Politics for a New Century, Lanham : Rowman and Littlefield, pp. 1-28.

PAGON M., VIRJENT-NOVAK B., DJURIC M., LOBNIKAR B. (1996), « European Systems of Police Education and Training ", in Pagon, M., Policing in Central and Eastern Europe : Comparing Firsthand Knowledge with Experience from the West, Ljublijana : College of Police \& Security Studies, pp. 551-574.

PETZOLD T., PICHL M. (2012), « Räume des Ausnahmerechts : Staatliche Raumproduktionen in der Krise am Beispiel der Bloccupy-Aktionstage 2012 », Kriminologisches Journal, no. 3, pp. 2011-227.

SCHMIDT-SEMISCH H. (2002), Kriminalität als Risiko, München, Gerling Akademie Verlag.

SCHROER M. (2013), « Raum, Zeit und Soziale Ordnung », in Ernst, P., Strohmaier, E., Raum, Konzepte in den Künsten, Kultur- und Naturwissenschaften, Baden-Baden, Nomos, pp. 11-24.

SMITH D. E. (1987), The Everyday World as Problematic. A Feminist Sociology, Boston, Northeastern University Press.

ULLRICH P. (2012), « Das repressive Moment der Krise », WZB-Mitteilungen, vol. 137, pp. 35-37.

WADDINGTON P.A.J. (1994), Liberty and Order. Public Order Policing in a Capital City, London, UCL Press.

\section{NOTES}

1. Même si les simulations ont lieu dans le monde " réel », nous les abordons ici non pas comme un donné objectif mais bien comme une production sociale. Les deux dimensions du réel et de la simulation sont des effets d'un processus de signification collective. Il existe une relation entre eux, qui représente la thématique centrale de cette contribution.

2. En dépit d'une inclination personnelle forte envers l'écriture inclusive, le masculin sera ici privilégié : cela permet d'attirer l'attention sur le fait que l'écrasante majorité des agents employés dans ce type de polices sont des hommes.

3. Il s'agit d'une protestation qui ne vise pas en premier lieu la communication et la médiation mais qui s'oppose de manière radicale aux conditions politiques existantes.

4. Une partie de la recherche a été soutenue par la recherche commandée par le Défenseur des droits à l'Institut national des hautes études de la sécurité et de la justice, «Désescalade de la violence et gestion des foules protestataires: quelle(s) articulation(s) en France et en Europe aujourd'hui? ».

5. La plupart des personnes employées dans cette profession étant des hommes, seules quelques femmes ont été interrogées.

6. Sur la simulation du maintien de l'ordre, voir Goreau-Ponceaud/Ponceaud-Goreau 2014 ; Kalalahti 2015/16; pour des informations générales sur la formation de base à Saint-Astier voir Bruneteaux 1996.

7. Pour cette distinction entre lieux dangereux et lieux en danger dans un contexte différent, voir Belina (2017).

8. Sans doute les positions sont-elles différenciées selon, par exemple, les forces de police impliquées, comme le montrent des positions parfois très favorables à la désescalade dans la 
gendarmerie française, par exemple in Général Véchambre, « Le maintien de l'ordre en France : sortir du discrédit », Revue de la Gendarmerie nationale, 267, 2020, p. 33-40.

\section{RÉSUMÉS}

Dans le monde entier, y compris en Europe, l'entrainement au maintien de l'ordre à l'aide de simulations réalisées au sein de villes artificielles s'est récemment développé. Les policiers s'y forment à ce qu'ils considèrent comme étant les futurs défis auxquels les forces de l'ordre seront confrontées. Dans cette perspective, ils élaborent des scénarios qui sont par la suite réalisés à l'aide d'importants moyens matériels et humains. Dans une perspective de sociologie urbaine et de sociologie politique, cet article étudie ces espaces de simulation de l'action policière en Europe. Il s'appuie sur une recherche ethnographique effectuée en Allemagne, en France, en Angleterre et en Irlande du Nord et pose la question de la convergence entre cette simulation et les évènements $d u$ "monde réel ». Le texte avance la thèse que ces formations ont pour caractéristique une politique du pire qui représente une tendance contemporaine partagée du maintien de l'ordre dans ces différents pays : ces formations présentant des situations exacerbées de danger et d'escalade de la violence, elles encouragent une représentation des manifestations comme des événements appelant un accompagnement de plus en plus resserré.

In a recent development, the policing of protest (demonstrations, rallies, etc.) is simulated worldwide, including in Europe, in mock cities. In those artificial settings police forces train what they anticipate as real challenges in protest policing. For this purpose, they develop scenarios that they implement with the extensive use of equipment and personnel. This article investigates these police simulations in Europe from an urban and political sociology perspective. It undertakes an ethnography of police simulations in Germany, France, England and Northern Ireland and raises the question of the convergence between simulation and reality. The text puts forward the argument that the trainings have as a characteristic a policy of the worst that adds to a contemporary trend in policing protest: as the trainings present demonstrations in an exacerbated way as situations of high danger and escalation, they encourage a social construction of demonstrations as events that call for a tough policing.

\section{INDEX}

Thèmes : Carnets de recherches

Keywords : Artificial cities, policing, police, Europe, training, simulation, apocalyptic scenario Mots-clés : Villes artificielles, maintien de l'ordre, police, Europe, entrainement, simulation, scénarios apocalyptiques 


\section{AUTEUR}

\section{ANDREA KRETSCHMANN}

Professeur de sociologie culturelle à l'Université Leuphana de Lunebourg, chercheuse associée au Centre Marc Bloch de Berlin.

andrea.kretschmann[at]leuphana.de 